\title{
Confidence: achievements and way forward
}

\author{
W. Raskob ${ }^{1, *}$, N.A. Beresford ${ }^{2}$, T. Duranova ${ }^{3}$, I. Korsakissok ${ }^{4}$, A. Mathieu ${ }^{4}$, M. Montero ${ }^{5}$, T. Müller ${ }^{1}$, \\ C. Turcanu ${ }^{6}$ and C. Woda ${ }^{7}$ \\ ${ }^{1}$ KIT - Karlsruhe Institute of Technology, Eggenstein-Leopoldshafen, Germany. \\ ${ }^{2} \mathrm{CEH}-\mathrm{UK}$ Centre for Ecology \& Hydrology, Lancaster, United Kingdom. \\ 3 VUJE - VUJE, a.s., Trnava, Slovak Republic. \\ ${ }^{4}$ IRSN - Institute for Radioprotection and Nuclear Safety, Fontenay-aux-Roses, France. \\ ${ }^{5}$ CIEMAT - Centro de Investigaciones Energéticas, Medioambientales y Tecnológicas, Madrid, Spain. \\ ${ }^{6}$ SCK-CEN - Belgian Nuclear Research Centre, Mol, Belgium. \\ ${ }^{7}$ HMGU - Helmholtz Zentrum München-German Research Center for Environmental Health, Neuherberg, Germany.
}

Accepted: 17 March 2020

\begin{abstract}
The project CONFIDENCE (COping with uNcertainties For Improved modelling and DEcision making in Nuclear emergenCiEs) final dissemination event attracted 88 participants to review and discuss the project results and provide ideas for future research work. The workshop highlighted progress in understanding uncertainties in all phases of an emergency. It was also demonstrated that consideration of uncertainties are important when developing countermeasure strategies. Stakeholder engagement as well as societal and ethical aspects in decision making have to be considered. Formal decision making tools were improved and tested. In addition, CONFIDENCE participants, representatives of international organisations and end users, provided their ideas on research needs and the way forward.
\end{abstract}

Keywords: emergency management / uncertainties / research needs / CONFIDENCE

\section{Introduction}

The CONFIDENCE project conducted a dissemination workshop in Bratislava, Slovak Republic. Organised by VUJE, three days from December 2-4, 2019 were devoted to review the progress made by presenting the research highlights and performing interactive demonstrations of the results. At the end of each day, the work package leaders provided their view on achievements and future research needs. On day four, representatives of international organisations, end users, the CONCERT and the CONFIDENCE coordinators provided their views too. This was completed by comments from the general audience of the meeting. This paper summarises these discussions from the dissemination workshop.

Individual contributors were:

- Eduardo Gallego (UPM, representing ICRP);

- Thomas Jung (BfS, representing CONCERT);

- Horst Monken-Fernandes (IAEA);

- Wolfgang Raskob (KIT, representing CONFIDENCE).

End users were represented by:

- Joanne Brown (IAEA, UK);

- Tom Charnock (PHE, UK);

*Corresponding author: wolfgang.raskob@kit.edu
- Petr Kuca (SURO, Czech Republic);

- Maarit Muikku (STUK, Finland);

- Paulo Nunes (APA, Portugal).

Platforms were represented by:

- Thierry Schneider (NERIS);

- Nick Beresford (ALLIANCE);

- Maria Antonia Lopez, Clemens Woda (EURADOS);

- Peter Mihok (SHARE).

CONFIDENCE work package leaders:

Irène Korsakissok (IRSN, France);

- Clemens Woda (HMGU, Germany);

- Nick Beresford (CEH, UK);

- Milagros Montero (CIEMAT, Spain);

- Catrinel Turcanu (SCK-CEN, Belgium);

- Tim Müller (KIT, Germany);

- Tatiana Duranova (VUJE, Slovak Republic).

Comments and suggestions listed below are not personalised but grouped thematically focusing on simulation models, stakeholders, societal and ethical aspects, decision making and operational use. 


\section{Main achievements}

\subsection{Simulation models}

The ensemble (realisation of a state by many simulations with slightly different initial conditions) approach for source terms and numerical weather data was implemented in all simulation models and decision support systems participating in CONFIDENCE. The usefulness of this approach was demonstrated in scenario calculations and the message to the operational community can be expressed as "Use ensembles! Trying to define a "worst case" a priori is not a good idea".

A new software tool for risk assessment using individual doses or doses to population groups and based on the WHO methodology was developed in CONFIDENCE. This allows for a fast assessment of risk taking into account the uncertainties of the input doses (e.g. from the ensemble calculations) and uncertainties of the risk assessment models. Using this tool, it is possible for the first time to use risk assessments in the early and transition phases of an emergency. For the Fukushima disaster for example, the first risk assessment was released only two years after the event, which is surely late.

The food chain and dose module FDMT of ARGOS (Hoe et al., 2002; Jacobsen et al., 2010) and JRODOS (Ievdin et al., 2010) has been analysed and a sensitivity analysis identified key parameters that contribute to the uncertainty of results. The database of the module was evaluated and those parameters that require modification identified. To complement this, soil-plant transfer data were collected for the Mediterranean environment.

Strengths and weaknesses of existing Cs process-based soil-plant models were investigated and a new approach for strontium developed. Comparing the new models with the FDMT showed that in the short-term (months-year) FDMT is appropriate, but in the long-term, process-based models are better able to identify "problem" areas and help to focus remediation measures. The inclusion of radioactive (or 'hot') particles in food chain modelling was investigated. In the short-term, accounting for the potential presence of radioactive particles in the soil is unlikely to be critical; in the longer term (decades), not accounting for particles in the deposit may underestimate ${ }^{137} \mathrm{Cs}$ and ${ }^{90} \mathrm{Sr}$ transfer to food products.

\subsection{Monitoring and data assimilation}

Uncertainties of monitoring devices (stationary, mobile) and monitoring endpoints (e.g. dose rate, air concentration) were defined, clearly stating, that spatial interpolation, site characteristics and iodine specification contribute most to the uncertainty of measured quantities, in particular gamma dose rates integrated over a short time period.

Data assimilation, combining measurements and model results was demonstrated as an important tool to obtain a consolidated radiological picture in phases where monitoring information is still sparse. Reconstruction of individual doses via a new tool and based on monitoring and spatial interpolation is an important step forward to comply with the European BSS that requests individual dose assessments.

A new method was tested that allows the use of electronic components $(>20 \mathrm{mGy})$ and memory cards $(>100 \mathrm{mGy})$ of irradiated smartphones to assess dose from external exposure. For internal exposure, a smartphone App for estimating the committed absorbed dose in the thyroid was developed. To complement this, uncertainties in thyroid dose monitoring were defined.

\subsection{Stakeholders}

CONFIDENCE has achieved progress in the development of sensible countermeasure strategies in inhabited and food production areas by involving relevant stakeholders. The uncertainties that play a role in the decision making process were identified, together with the main criteria/attributes used for decision making on countermeasure strategies in the transition phase. The importance of generic and site specific scenarios were highlighted together with the added value of stakeholder panels as key elements for decision making. A categorisation of the important uncertainties was developed together with recommendations on how to implement and improve the process of developing countermeasure strategies in the operational context. Preferences and expectations were collected with Delphi studies and within scenario-based workshops providing a sound basis for application of formal decision aiding methods.

\subsection{Societal and ethical aspects}

The analyses of past accidents as well as observations of emergency exercises were used in identifying societal uncertainties in emergency and post-accident situations. Mental models were developed to better understand the behaviour of the general public and their sense making in an emergency. Identifying uncertainties was one of the key aspects of this work activity. The quantitative analysis of surveys in three countries, Spain, Belgium and Slovenia, were used to identify key problems in communication of recommendations by authorities to the public. A framework for dignified living conditions has been developed and tested in stakeholder panels. Most important was the conceptualisation and identification of social uncertainties that can help to develop better recommendations in future. Finally, advice was developed for improving the robustness of emergency preparedness and response and for enhancing capacities of local actors.

\subsection{Decision making and communication}

Communication approaches including visualisation of maps, indicators of result uncertainties, smartphone Apps, text messages, numerical, narrative or mixed messages and video were tested. This resulted in the development of advice on communication of uncertainties and the use of communication tools in preparedness and response, for both decision makers and the general public.

An agent-based model was developed to simulate and better understand collaborative decision making in nuclear emergencies. Various negotiation schemes were developed and tested to understand the difference between voting and consensus seeking schemes. The MCDA tool was enhanced 
to include uncertainties from the simulations but also uncertainties in the preferences and weights of the decision maker. The application in scenario-based stakeholder workshops demonstrated the applicability of the enhanced tool.

\subsection{Operational use}

The many simulation models developed were at least partly implemented and thus available for the end users. Operational guidelines for the use of the new tools and methods were developed allowing their further testing under operation conditions.

\subsection{Education and training}

One training course for decision makers and stakeholders was developed as guidance on the use of uncertainty information by decision makers at the various levels within the decision making process. A further training course/ workshop was prepared for students and professionals transferring knowledge from derivation and application of process-based models to predict radionuclide activity concentrations in foodstuffs. A series of lectures and accompanying material were developed focusing on building of capabilities, trust and confidence in radiation protection issues (engage the young generation).

\section{Research needs}

\subsection{Simulation models}

The application of the ensemble approach in simulation models requires further testing and clarification. In particular, proper source term ensembles have to be defined. The meteorological ensembles that are appropriate for operational ensembles have to be further refined, in particular to what extent can automated clustering approaches be applied in reducing the number of runs needed to represent uncertainty. As resources are limited, different types of dispersion models - Gaussian versus Lagrangian particle models - should be compared to identify the model needs for uncertainty estimation. In particular, the question has to be addressed if simpler models (less computation time) with more meteorological ensembles represent uncertainties as well as complex models (high computation time) with a limited set of meteorological ensembles.

There was controversial discussion of the risk model. The application of the model as an indicator for medical screening was recognised, whereas the application for decision making in the early phase was questioned. One recommendation was to apply and test the approach in the operational community to identify its strengths and weaknesses.

Process-based radioecological models were judged as important for longer-term planning. However, some requirements to further test and/or develop the models were identified. The weakness of some model (empirical) databases was acknowledged and data collection encouraged (e.g. arid environments) and databases need to be kept up to date. The question was raised as to whether international organisations such as the IAEA can be involved in this (e.g. revision of TRS472 [IAEA, 2014]). With the new emerging process-based models, clear guidance should be developed as to when and under which conditions simple or complex models should be applied. Future research priorities for human food chain modelling are presented in Beresford et al. (2020).

\subsection{Monitoring and data assimilation}

The simulation model for individual dose reconstruction requires further testing for scenarios with little information on the nuclide vector and sparse monitoring information. The estimation of the internal exposure in dose reconstruction requires also further research. This is also the case for the data assimilation approach, which very much relies on information on the nuclide vector. The methodology also depends on the number and location of monitoring stations, which has a direct impact on monitoring strategies. This requires a more holistic view on modelling and monitoring in future. Information from citizen monitoring/citizen science should be included in modelling as this information will become available and should be considered for decision making. Research is needed to judge the quality of monitoring data, estimate the uncertainty and how to combine the monitoring data with operational information to obtain a complete operational picture using all the monitoring information available. The integration of biodosimetrical monitoring into emergency management still requires further investigation.

\subsection{Stakeholders}

Within CONFIDENCE, many recommendations for stakeholder engagement and strategy development were prepared. The challenge is now to implement them. This requires further research and testing in European Member States. CONFIDENCE attracted mainly stakeholders from authorities and public organisations but did not include NGOs or industry. This should be considered in further projects. It was also controversially discussed, to which extend experience from natural disasters could be used in our domain. This should be clarified in a research project.

\subsection{Societal and ethical aspects}

CONFIDENCE has highlighted many aspects of societal and ethical uncertainties in decision making. An area that requires further research is the question how to strengthen resilience of the society in an emergency. This might be realised by encouraging citizens to support first responders. Vulnerability assessment might also help in identifying ways to increase resilience. Societal and stakeholder networks were regarded as extremely valuable and therefore effort should be spent to keep them alive. Further work on mental models or means to better understand the public is important for preparedness and response to target messages in a way that the general public understands and follows. Citizen science was discussed from many different viewpoints and a final recommendation from the panel at the last day of the workshop was that it should be considered as a valuable resource and projects should be prepared to discuss this further. 


\subsection{Decision making and communication}

The agent-based model was regarded as interesting, and its capabilities should be explored in a larger project in future. The MCDA tool should be further tested and applied in other projects and in the operational context. Communication was judged as very important and CONFIDENCE demonstrated that more research should be carried out on several aspects such as visualisation of results, communication of the quality of results, communication of negligible doses, how to prepare information in advance and identify which tools are best for which situation. This should also include all aspects of social media.

\subsection{Operational use}

The operational community highly appreciated the operational aspect of CONFIDENCE, in particular that prototypes of new models become an integral part of decision support systems or atmospheric dispersion models. The end users also requested that everybody should have access to these tools and that they should be maintained. They also requested more customisation work to adapt these new tools to national and even local conditions - important e.g. for the radioecological models. Ensemble modelling should be further tested in the operational environment and the use of the health risk model should be further explored. Training of operators and decision makers in the new tools is required as well as in the application and testing of the new communication approaches.

\subsection{Education and training}

Education and training was judged as essential for dissemination of results from research projects. Training for the operational community should be considered as part of the activities of existing Radiation Protection platforms. The NERIS platform was encouraged to consider the training course on uncertainty in decision making as complementary to the basic course on emergency management.

\section{Discussion and conclusions}

CONFIDENCE has developed many new tools, approaches and recommendations for their use and implementation. Even though CONFIDENCE is a research project, some of the end products will become part of operational tools. However, this is limited to some of the simulation models and the MCDA toolkit. The whole wealth of results with the many recommendations from WP4 to WP6 on stakeholders, societal and ethical aspects and decision making could not fully be explored within the project. Even for the ensemble modelling, the operational recommendations are only a starting point for future discussion. To explore this further, a so-called "Large Scale Demonstration Project as funded under other frame work programs (see e.g. EDEN project https://eden-security-fp7.eu/eden,id,11,about.html) could demonstrate the applicability of our approaches to become operationally applicable all over Europe.
The objective of these demonstration projects is to bridge the gap between stakeholder need/expectation, existing tools and research results and to evaluate the best possible solution for operational use. The aim is not directed towards research - even if research is always part of such a project- but towards the demonstration of the methods/tools in an operational environment. Research is needed to tailor some of the solutions such that they are fit for purpose for such demonstrations. As a result of this, methods and tools will be identified that are fit for purpose and/or which require further research/development to reach such a status.

In addition to such a large-scale demonstration project, future research on uncertainties is required. CONFIDENCE identified key areas, but as discussed in Section 3 above, many aspects still require further work. This was acknowledged by the representatives of the European Research platforms ALLIANCE, EURADOS, MELODI, NERIS and SHARE. The findings of CONFIDENCE and identified research requirements have been used to draft the revised Strategic Research Agendas of the platforms (Salomaa, 2019). This hopefully assures some continuity in European research.

Finally, the many developers of the simulation models, methods and recommendations will continue working on their "babies" to meet the expectations of end users. Emergency management - different from basic research - always has the operational aspects that need consideration to be better prepared for the next disaster that will happen for sure.

\section{Acknowledgement}

The work described in this paper was conducted within the CONFIDENCE project, which was part of the CONCERT project. This project has received funding from the Euratom research and training programme 2014-2018 under grant agreement No. 662287.

Disclaimer (Art. 29.5 GA). This publication reflects only the author's view. Responsibility for the information and views expressed therein lies entirely with the authors. The European Commission is not responsible for any use that may be made of the information it contains.

\section{References}

Beresford NA, Barnett CL, Chaplow J, Lofts S, Wells C, Brown JE, Hosseini A, Thørring H, Almahayni T, Sweeck L, Guillén J, Lind O-C, Oughton DH, Salbu B, Teien H-C, Perez-Sánchez D, Real A. 2020. CONFIDENCE Overview of improvements in radioecological human food chain models and future needs. Radioprotection 55(HS1). https://doi.org/10.1051/radiopro/2020019.

Hoe S, Müller H, Gering F, Thykier-Nielsen S, Havskov Sorensen J. 2002. Argos 2001 A decision support system for nuclear emergencies, American Nuclear Society Transactions. WinterMeeting 87: 574-579.

Jacobsen LH, Andersson KG, Charnock T, Kaiser JC, Gering F, Hoe SC, Juul Larsen L. 2010. Implementation in ARGOS of ERMIN and AGRICP. Radioprotection 45(5): S191-S198.

IAEA. 2014. Handbook of parameter values for the prediction of radionuclide transfer to wildlife. Technical Reports Series No. 479. Vienna: IAEA. Available from: http://www-pub.iaea.org/ MTCD/Publications/PDF/Trs479_web.pdf. 
Ievdin I, Trybushnyi D, Zheleznyak M, Raskob W. 2010. RODOS reengineering: aims and implementation details. Radioprotection 45 (5 Suppl.): S181-S189.
Salomaa S, Ed. 2019. Updating the SRAs of MELODI, ALLIANCE, NERIS, EURADOS and EURAMED. CONCERT Deliverable D2.13. Available from: https://concert-h2020.eu/en/Publications.

Cite this article as: Raskob W, Beresford NA, Duranova T, Korsakissok I, Mathieu A, Montero M, Müller T, Turcanu C, Woda C. 2020. Confidence: achievements and way forward. Radioprotection 55(HS1): S39-S43 